\title{
AN ELECTROMAGNETIC DETECTOR FOR RELIC AXIONS
}

\author{
Donald E. Morris \\ Lawrence Berkeley Laboratory \\ University of California \\ Berkeley California 94708
}

May 1984

\section{DISCLAIMER}

This report was prepared as an account of work sponsored by an agency of the United States Government. Neither the United States Government nor any agency thereuf, nor any of their employees, makes any warranty, express or implied, or assumes any legal liability or responsibility for the accuracy, completeness, or usefulness of any information, apparatus, product, or process disclosed, or represents that its use trould not infringe privately owned rights. Reference herein to any specific commercial product, process, or service by trade name, trademark, manufacturer, or otherwise does not necessarily constitute or imply its endorsement, recommendation, or favoring by the United States Government or any agency thereof. The views and opinions of authors expressed herein do $r$. necessarily state or reflect those of the United States Government or ny agency thereof.

"This work was prepared for the US Department of Energy under Contract Number DE-AC 03-76SF00098" 
An Electromagnetic Detector for Relic Axions

\author{
Donald E. Morris
}

\begin{abstract}
Axions are particles of small nuass postulated to explain CP conser ration in strong interactions. The predicted properties of axions provide an explanation for the early clustering of matter into galaxies, the mass in galactic halos, and the missing mass sufficient to close the universe. The interaction of axion with ordinary matter would be extremely weak, but axions should be detec able, because in a strrng magnetic field relic axions of mass $\mathrm{m}_{\mathrm{a}}$ would conver into microwave photoi vith frequency $f=m_{a} c^{2} / h$. Predictions for the frequ ncy range from 3 and $2 \leqslant h z$ for relic axions which provide mass density sufficient to close the universe.

We propose a laboratory search for relic axions. A specific experimental apparatus is described and system performance is estimated to illustrate the design principles. The microwave signal from axion conversion is produced in a $\mathbf{3 0}$ liter microwave cavity which contains dielectric phase shifting plates to give a quality factor ( $Q$ ) greater than $10^{6}$, and to provide the correct phasing of the microwave electric field throughout the cavity. The cavity is placed in an 8 Tesla superconducting magnet, and the signal is measured with a conventional microwave receiver employing a GaAs FET RF amplifier or Schottky diode mixer. The cavity, magnet and $R F$ amplifier/mixer will operaie at $4^{\circ} \mathrm{K}$, with noise temperature between $20^{\circ} \mathrm{K}$ and $200^{\circ} \mathrm{K}$. The system is equally effective in principle at all frequencies between $1 \mathrm{GHz}$ and $100 \mathrm{GHz}$ at which microwave receivers are available. The corresponding range of axion mass which can be covered is $4 \times 10^{-6} \mathrm{eV}$ to $4 \times 10^{-4} \mathrm{eV}$. A search can be carried out over an octave of frequency in an observation time of three months with sufficient sensitivity to detect axions if they make up the Galactic halo.
\end{abstract}


(ii)

\section{Contents}

Introducton 1

Signal and Measurement Time $\quad 2$

Design of Experiment 3

Experimental Apparatus 3

A. Cavity Design 3

B. Magnet 4

C. Microwave Receiver 4

Measurement Strategy $\quad 4$

A. False Alarms

1. Statistical Fluctuations $\quad 5$

2. Magnetic Resonance 5

3. Standing Waves in Receiver System 5

B. Correlation Detection 5

System Performance 6

$\begin{array}{ll}\text { Conclusions } & 6\end{array}$

$\begin{array}{ll}\text { Acknowledgements } & 6\end{array}$

References $\quad$ ?

$\begin{array}{ll}\text { Appendix I: } & \begin{array}{l}\text { Improvement of Cavity } Q \text { by Quarter } \\ \text { Wave Dielectric Plates }\end{array}\end{array}$

Appendix II: $\quad$ Optimum Phasing of Electric Field

Using Half Wave Dielectric Plates 9

Appendix III: $\quad$ Field Patterns and Wall Losses in

Cavities with Dielectric Plates

Appendix IV: $\quad$ Mode Dependence of Effective Volume of a Cavity for Axion Detection 13

Figures 1-6 


\section{Introduction:}

The axion provides the best explanation of CP conservation in strong interactions (Peccei and Quinn 1977, Weinberg 1978). Axions with very small mass ( $m a$ between $10^{-5} \mathrm{eV}$ and $10^{-3} \mathrm{eV}$ ) can explain the clustering of matter in the early universe into galaxies and account for the mass of galactic halos (Sikivie 1982)(Ipser \& Sikivie 1983). Axions may constitute a large fraction of all matter and provide sufficient mass density for closure of the universe (Stecker \& Shafi 1983). An upper limit for the axion mass of $10^{-2} \mathrm{eV}$ has been estimated astrophysically by the increase of energy loss in red giant stars that axion production would provide and the unacceptable consequences for stellar evolution(Dicus et al 1980). The axion mass has a lower limit of $10^{-5} \mathrm{eV}$, since if it were smaller the mass density of axions produced in the early unimerse would far exceed the critical density (Preskill,Wise and Wilczek 1983; Abbot \& Sikivie 1983; Dine \& Fischler 1983). Abbot \& Sikivie estimated an axion mass of about $10^{-4}$ ev (within a factor of three) as providing the critical mass density of the universe. The corresponding microwave frequency is about $24 \mathrm{GHz}$.

Sikivie(198.3) pointed out that very light axions can be detected by their conversion into photons in the presence of a strong static magnetic field. One of his proposed detection schemes employs a microwave cavity in a uniform static magnetic field, and is suitable for a search for axions in the galactic halo with mass near $10^{-5} \mathrm{ev}$.

Using a special cavity design, a search can be carried out over the range of axion masses from $4 \times 10^{-6} \mathrm{eV}$ to $4 \times 10^{-4} \mathrm{eV}(1 \mathrm{GHz}-100 \mathrm{GHz}$ ), which includes all of the range of axion mass which could provide the critical mass density of the universe, and most of the range which could provide the mass of the galactic halo.

Sucessful detection of axions would have far reaching consequences in particle physics and in cosmology and astrophysics. A negative result from this axion search would contradict the hypothesis of the contribution of axions to missing mass of galactic halos and to formation of galaxies in the early universe, and the contribution of axions to the $c_{r}$ ssure mass density of the universe. 


\section{Signal and Measurement Time}

Axions in the halo of our galaxy will interact with a strong static magnetic field and convert into photons in a suitable cavity. The powew from axion conversion will be small compared with the noise power from the cavity and a receiver connected to the cavity, but can be detested by signal averaging for a sufficient time and comparing with the noise power at adjacent frequencies. Sikivie(1983,1984) has calculated the power due to axion conversion into photons in a TM mode of a cavity in a static magnetic field $B_{0}$ as:

$$
\begin{array}{r}
P_{a}=(1 / 4 \pi) \cdot\left(3 \times 10^{-18} \mathrm{Watt}\right) \cdot\left(\mathrm{V} / 10^{5} \mathrm{~cm}^{3}\right) \cdot\left(\mathrm{B}_{\mathrm{o}} / 10 \mathrm{Tesla}\right)^{2} \\
\cdot\left(\mathrm{m}_{\mathrm{a}} / 1.24 \times 10^{-5} \mathrm{eV}\right) \cdot\left(1 / \mathrm{n}^{2} \mathrm{~m}^{2}\right)
\end{array}
$$

where $V$ is the volume of the cavity, $B_{0}$ is the value of the static magnetic field in the cavity in Tesla, $m_{a}$ is the mass of the axion, $n$ and $m$ are the mode numbers of the microwave field in the cavity. The factor $(1 / 4 \pi)$ is a correction given by Sikivie(private communication).

The expected bandwidth $B$ of the photons from axion conversion is about $10^{-6} \mathrm{f}$, due to the kinetic energy of the axions at the velocity of galactic matter $v_{\mathrm{g}}$ , which is about $10^{-3} c\left(B=f-m_{a} c^{2} / h=1 / 2\left(v_{g} / c\right)^{2} f\right)$. For best sensitivity the cavity coupling is adjusted for a cavity loaded $Q$ of $10^{6}$ and the receiver should measure the power in a corresponding bandwidth. Axion photon conversion is coherent, so the power is proportional to cavity $Q$ until the bandwidth of the cavity is less than the bandwidth of the axion signal. In the following expressions we approximate the axion frequency distribution by a gaussian with an assumed bandwidth of $10^{-6} f$ and take $P_{a}$ proportional to the product of the axion frequency distribution and the cavity response curve.

We can calculate the temperature of this signal: $T_{a}=P_{a} / k B$. The axion bandwidth $B 10^{-6} f$, and $k$ is Boltzmanns constant. If $n=m=1$, then

$$
I_{a}=\left(5.7^{\circ} \mathrm{Kelvin}\right) \cdot\left(\mathrm{V} / 10^{5} \mathrm{~cm}^{3}\right) \cdot\left(\mathrm{B}_{\mathrm{o}} / 1 \mathrm{C} \mathrm{T}\right)^{2} \cdot\left(\mathrm{Q} /\left(\mathrm{Q}^{2}+10^{12}, 1 / 2\right)\right.
$$

$T_{a}$ is independent of the axion rest mass frequency (it $n=m=1$ ), and so this detection system can be used over a wide range of axion mass. The measuring 
time for a specified signal to noise ratio

$$
t_{m}=(S / N)^{2} \cdot\left(T_{r} / T_{a}\right)^{2} \cdot\left(10^{6} / 2 f\right)
$$

The factor $10^{6} / 2 \mathrm{f}$ is introduced since $2 \mathrm{~B}$ independent measurements can be made each second, and $B \quad 10^{-6} \mathrm{f}$. The rate of measurement $1 / t_{m}$ is proportional to $B^{4} V^{2} f / T_{r}{ }^{2}$ (for cavity $Q_{c}$ greater than $10^{6}$ ). So the measurement time will be independent of the axion rest mass frequency if the receiver noise temperature $T_{r}$ increases with frequency roughly as $\mathrm{f}^{1 / 2}$. The measurement time $t_{m}$ must not exceed a few seconds for each frequency to permit a sequential search of a wide frequency range in a reasonable time.

\section{Design of Experiment}

\section{Experimental Apparatus}

The apparatus consists of a specially designed cavity, a superconducting magnet, and a GaAs FET amplifier (or Shottky diode mixer above $20 \mathrm{GHz}$ ), all at $4^{\circ} \mathrm{K}$. The output of the amplifier/mixer is fed to a conventional microwave receiver. The system is shown in figure 1 .

Cavity Design: For adequate sensitivity we will require a cavity design which allows a very high $Q$, has a large effective volume in which the axion to photon conversion takes place coherently, and is tuneable over a wide range to limit the number of cavities required. These objectives can be achieved with a cavity design employing dielectric phase shifting plates. The dielectric may be aluminum oxide caramic with $\mathrm{K}=10$, or preferably sapphire, which has $\mathrm{K}=13$ and a loss tangent of $10^{-9}$ at $4^{\circ} \mathrm{K}$. In the example used for calculation of system performance, the cavity is rectangular with dimensions $25 \mathrm{~cm} \times 30 \mathrm{~cm} \times 70 \mathrm{~cm}$. and will contain a stack of halfwave dielectric plates with overall dimensions $25 \mathrm{~cm} x$ $25 \mathrm{~cm} \times 50 \mathrm{~cm}$ The unloaded $Q$ of the cavity will be increased to at least $5 \times 10^{6}$ by quarter wave dielectric plates (see figurt 2, and Appendix I).

Half wave dielectric phasing plates will be employed to give a frequency independent effective cavity volume equal to about half the geometric volume (Appendix II). The cavity will be tuned over a half decade frequency range by varying the width of the cavity and the spacing between the plates. The thickness of the plates need not be exactly $/ 2 \mathrm{~K}^{1 / 2}$ so long as the spacing is nearly 
uniform. By stacking the plates in pairs, or substituting plates of different thickness, other frequency ranges can be covered. Fine tuning will be accomplished by varying the cavity width by piezoelectric transducers or by moving sheets of a low loss dielectric such as Teflon between regions of high and low electric field.

Magnet: The signal temperature is proportional to the stored energy of the static magnetic field within the cavity (equation 2). A cost effective design with $\mathrm{B}_{\mathrm{O}}=8$ Tesla will permit use of a $\mathrm{VbTi}$ alloy superconductor at $4^{\circ} \mathrm{K}$. Only one magnet is required to cover the entire frequency range. We propose to use an 8 Tesla magnet of proven design with clear bore of $38 \mathrm{~cm}$ and reasonably uniform field over $50 \mathrm{~cm}$ length.

Mictowave Receiver: The receiver will use cooled GaAs FET amplifiers up to $20 \mathrm{GHz}$, cooled to $4^{\circ} \mathrm{K}$ to give a noise temperature ranging from $10^{\circ} \mathrm{K}$ at $1 \mathrm{GHz}$ to $100^{\circ} \mathrm{K}$ at $20 \mathrm{GHz}$. Above this frequency, GaAs Shottky diode mixers with GaAs FET IF amplifiers cooled to $4^{\circ} \mathrm{K}$ can give noise temperatures below $200^{\circ} \mathrm{K}$ up to $100 \mathrm{GHz}$ (Lubin et.al. 1983). A considerable number of microwave receiver systems will be required to cover the wide frequency range in which axions may be found, especially above the range at which broadband receivers are available.

\section{Measurement Strategy}

It will be necessary to locate and adjust the cavity resonance frequency and coupling. To accomplish this, the cavity is first excited by a loosely coupled sweep signal generator and the cavity resonant frequency and loaded $Q$ are determined by measurement of the signal by the microwave receiver. The cavity resonance is adjusted to the desired frequency, and receiver coupling is adjusted for optimum loaded $Q$, both by mechanical servos. Then the signal generator is switched off. Because the expected axion signal power is much smaller $\left(10^{-2}\right)$ than $t$ ae receiver noise power, special precautions will be needed to reduce systematic errors to acceptable levels. For example, the resonance frequency can be mechanically modulated at a low frequency by transducers on the cavity walls or by moving a dielectric in the cavity between regions of high and low electric field. A multiplex receiver will permit observaticn of the noise power in a number of adjacent frequency channels with outputs gated while the resonance is swept between them at the modulation frequency. The axion signal is an excess of power in cre of the channels which does not change with tuning of the cavity. The 
power in the adjacent channels is compared to cancel drift and systematic errors. After a suitable measurement time the resonance frequency is readjusted using the sweep signal generator, and measurements are made in another set of channels. A computer will carry out the measurement sequence and log the data.

Systematic errors must be reduced to a low level since the axion signal of $0.4^{\circ} \mathrm{K}$ is much smaller than the receiver noise. Cosmic microwave background signals have been measured to millidegree levels, which are two orders of magnitude smaller than required for this experiment, but the system bandwidth was much larger and no cavities were involved (Lubin et al 1983).

False Alarms: Several sources of false alarms and spurious signals must be dealt with.

Statistical fluctuations will cause many false alarms because of the low $\mathrm{S} / \mathrm{N}$ ratio of $3 / 1$. Since the axion signal is always present, an apparent signal can be verified by repeated the measurement at the same frequency.

Magnetic resonance such as cyclotron resonance in the cavity walls may be excluded by changing the magnetic field.

Standing waves and resonances in the microwave conponents and transmission line and in the RF amplifier may give noise peaks in the spectrum. Any axion signal will disappear when the static magnetic field is turned off, but this is not a conclusive test, since the resonances and standing waves may change with magnetic field. An alternative test is to retune the cavity on the order of $1 \%$ so that an even mode ( $m=2$ for example) is tuned to the frequency at which the noise peak was found. This is done without disturbing or retuning the microwave components or receiver or changing the static field. Since the axion photon conversion is $z$ ero for even modes, the signal should disappear.

Correlation Detection: The existence of an axion signal can be verifjed by correlation detection. Because of the large wavelength of the axions, two cavities tuned to the same frequer $y$ and placed close together (less than $10^{3}$ photon wavelengths) will have correlated outputs. After passing through independent amplifiers and hetrodyning with the same local oscillator to a convenient low frequency, the two signals can be multiplied, and the product compared with the individual signals to determine the correlation. So long as the two cavities and receivers are sufficiently isolated from each other there will be no correlation between ineir outputs except from axion signals. 


\section{System Performance}

From equation 2, the signal temperature will be $0.4^{\circ} \mathrm{K}$. From equation 3 , with a receiver noise temperature of $30^{\circ} \mathrm{K}$ at $3 \mathrm{GHz}$, the measurement time at each frequency is 10 seconds for a $3 / 1 \mathrm{~S} / \mathrm{N}$ ratio. At $90 \mathrm{GHz}$ a receiver noise temperature of $200^{\circ} \mathrm{K}$ will require a measurement time of 15 seconds. Therefore, the operating time to scan an octave of frequency will be about three months, with sensitivity sufficient to detect axions which constitute the Galactic halo.

Once the mass of the axion is precisely known, the same detector can easily determine the spectrum of axion kinetic energies and observe the doppler shift due to the motion of the earth, since relatively few narrow channels must be measured. For example, the same cavity, magnet system and receiver could be connected to a 100 channel multiplex spectrum analyrer with channel width and spacing $=10^{-8} \mathrm{f}$ to give a $10 / 1 \mathrm{~S} / \mathrm{N}$ ratio in each channel in three hours running time.

\section{Conclusions}

An axion detector is described which should be capable of detecting axions which make up the Galactic halo. No new technology is required to be developed in order to make the experiment feasible, since specifications of both the magnet and mircrowave receivers are based on proven technology, and established performance levels. It will be necessary to demonstrate that the proposed cavity design can achieve the specified $Q$ and field patterns, and that cavity tuning and coupling can be controlled adequately. Because the expected signal power is much smaller than the receiver noise power, systematic errors will have to be reduced to low levels by modulation and comparison techniques.

\section{Acknowlegements}

I wish to thank Philip Lubin and George Smoot for advice regarding the performance of microwave receivers for small signals and for editorial suggestions, Shane Burns for explanation of theory, and Carl Pennypacker for stimulating my interest in detection of axions. Thanks are due to Pierre Sikivie for several stimulating discussions about the properties of relic axions and their detection. 


\section{References:}

A bbot,L.F., and Sikivie,P. 1983, PL120B,133

Dicus,D.A.,Kolb,E.W.,Teplitz,V.L., \& Wagoner,R.v. 1980, Phys RevD22,839

Dine, M., and Fischler,W. 1983, PL120B,137

Ipser,J., and Sikivie,P. 1983, PRL50,925

Lubin,P.M.,Epstein,G.L., and Smoot,G.F. 1983, PRL50,616

Peccei,R.D., and Ouinn,H.R. 1977, PRL38,1440; Phys RevD16,1791

Preskill,J., Wise,M.B., \& Wilczek,F. 1983, PL120B,127

Sikivie,P., 1982, PRL48,1156

Sikivie,P., 1983, PRL51,1415

Sikivie,P., 1984, PRi52,

Stecker,F.W., and Shafi,Q. 1983, PRL50,928

Weinberg,S. 1978, PRL40,223 


\section{Appendix I}

\section{Improvement of Cavity Quality Factor by Quarter Ware Dielectric Plates}

The unloaded $Q$ of an aluminum rectangular cavity $25 \times 25 \times 60 \mathrm{~cm}$, cooled to $4^{\circ} \mathrm{K}$ would be on the order of $5 \times 10^{5}$. (a) The required $Q$ is larger than $5 \times 10^{6}$ so that most of the signal will reach the receiver instead of being absorbed in the cavity. To increase the cavity $Q$, quarter wave plates spaced $\lambda / 4$ from the cavity walls are used as impedance transformers to increase the mismatch and reduce the energy density at the cavity walls. The idea is illustrated in figure 2 which shows the equivilen: transmission line circuit. The effective surface impedance and therefore the wall loss can be reduced by a factor $K$, with corresponding increase in $Q$. The $E$ and $H$ fields and distributions of the wall losses are given in Appendix III and figures 3 and 4, and it is shown that only two side walls need be covered by quarter wave plates since the losses in the other two cavity side walls will be small in the appropriate mode. Magneto-resistance will not increase the losses in walls which are parallel to $\mathrm{B}_{0}$ since the microwave currents flow parallel to $\mathrm{B}_{\mathrm{O}}$ in TM modes, such as the mode of interest. Losses in end walls perpendicular to $B_{0}$ will be small if the cavity is elongated in the direction of $B_{0}$.

(a) The surface resistance of pure aluminum (RRR=450) at $4^{\circ} \mathrm{K}$ in the anomolous skin effect limit $Z_{s}=10^{-9} \mathrm{f}^{2 / 3} \mathrm{ohm} /$ square, where $\mathrm{f}$ is in $\mathrm{Hz}$.

(See Progress in Cryogenics, Vol 4, 1964 page 134) The cavity will have $Q=\left(Z_{0} / Z_{s}\right)(a / \lambda)$ where $a$ is the width of the cavity, and $z_{0}=377 \mathrm{ohm} / \mathrm{square}$ (the impedence of free space). For $a=25 \mathrm{~cm}, Q=314 \mathrm{f}^{1 / 3}$, so that $Q=4.54 \times 10^{5}$ at $3 \mathrm{GHz}$ and $1.2 \times 10^{6}$ at $60 \mathrm{GHz}$.

(b) End wall losses may be further reduced by placing them several wavelengths from the ends of the $\lambda / 2$ dielectric plates described in the next section, since the EM field will fall off exponentially outside the stack. (The mode of interest can be decomposed into plane waves travelling almost normal to the dielectric plates, and the average velocity in the stack is reduced by a factor $\left(1 / 2+1 / 2 \mathrm{~K}^{1 / 2}\right)$, resulting in total internal reflection.) 
Appendix II

\section{Optimum Phasing of Electric Field Using Half Fave Dielectric Plates}

In equation (2) we have taken $n=m=1$. In that ense $(2 / \lambda)^{2}=1 / a^{2}$ $+1 / b^{2}+1 / d^{2}$ where $a, b$ and $d$ are the dimensions of the rectangular cavity. This will be satisfactory only when $\lambda$ in in the order of the cavity ditnensions. The amplitude for axion photon conversion is proportional to the integral of $\mathrm{E}$ ' $\mathrm{B}$ over the cavity volume when the phase of the axion field is the same throughout the cavity, and this is the case for the non-relativistic halo axions since the axion wavelength is $10^{3}$ larger than that of the photon $\left(\lambda_{a}=h / p=h / m c \beta_{\alpha}=10^{3} \lambda_{\gamma \gamma}\right)$. The integral of $E \cdot B$ is reduced when $E$ does not have the same phase throughout the cavity. The result for a rectangular cavity is giver in Appendix IV. Because of the small spread in axion energy $\rightarrow 10^{-6} \mathrm{f}$, the phase remains coherent for $10^{6}$ cycles, and this is the basis for the use of high cavity $Q=10^{6}$ to enhance the power of axion conversion into photons.

The microwave $\mathrm{E}$ field is required to be parallel to $\mathrm{B}_{\mathrm{O}}$, along the axis of the magnet. Since EM waves are transverse, we will have standing waves transverse to the magnet axis, and the phase of the $E$ field will reverse after every half wavelength. The solution is to arrange for an additional phase reversal $c \hat{i} \mathrm{E}$ after every half rave so that $E$ will be in phase throughout the whole cavity, then the $1 / \mathrm{n}^{2} \mathrm{~m}^{2}$ term in eq 1 will drop out. The phase reversal can be provided by metal baffles in the cavity or by cielectric plates.

Metal Plates spaced every $\lambda / 2$ with slots along the $3_{0}$ dirstion (figure 6 ) will reverse the phase of $E$ in successive compartments. This snethod has several disadvantages: 1. the cavity $Q$ is reduced at higher frequencies as the spacing of the baffles becismes smaller, 2. the spacing of the partitions is critical since the resonance frequencies of the cavity sections must be nearly identical, 3 . the resonance frequencies of the cavity sections must track very accurately as the resonance frequency is varied and so this complex structure would be difficult to tune over a wide sange. All of these disadvantages are avoided in the design earyloying dielectric phase shifting plates.

The Dielectric plates are arranged in the cavity with their surfaces parallel to the $B_{0}$ dizerticn and spaced with $\lambda / 2$ separaticn (figure 5 ). The thickmess of the plates will be $\mathrm{X} / 2 \mathrm{~K}^{2 / 2}$, where $\mathrm{K}$ is the dielectric censtant, and the field 
inside the dielectric $E^{\prime}=E_{d} / K^{1 / 2}$ (see Appendix I). For sapphire, $K=13$, and the effective volume of the cavity is 0.52 of the geometric volume after accounting for the space taken by the dielectric plates and subtraction of the integral of $E^{\prime}$ with the wrong phase in the volume inside the plates.(Appendix IV B). Sapphire has a lass tangent on the order of $10^{-9}$ at $4^{\circ} \mathrm{K}$ so dielectric losses are neglegible. 


\section{Appendix III}

\section{Field Patterns and Wall Losses in Cavities with Dielectric Plates}

\section{A. Fỉeld Patterns}

1. Cavity with half wave dielectric plate. ( see figure 3 )

For the lowest TM mode $E_{x}=E_{y}=H_{z}=0$,

$$
\begin{aligned}
& \text { for }-a(x<0, \\
& E_{z}=E_{0} \sin (\pi x / a) \sin (\pi y / b) \\
& H_{x}=-j\left(E_{o} / c\right)(\lambda / 2 b) \sin (\pi x / a) \cos (\pi y / b) \\
& H_{y}=j\left(E_{o} / c\right)(\lambda / 2 a) \cos (\pi x / a) \sin (\pi y / b)
\end{aligned}
$$

And imposing the boundary conditions at $x=0: H_{x}=E_{z}=0$ and $H_{y}=H_{y}{ }^{\prime}$. (since $\nabla \times H=\partial D / \partial t=K \partial E / \partial t=0$ because $E_{x}=E_{y}=E_{z}=0$ ) Then for $0<x<a K^{-1 / 2}$

$$
\begin{aligned}
& E_{z}{ }^{\prime}=K^{-1 / 2} E_{o} \sin \left(K^{1 / 2} \pi x / a\right) \sin (\pi y / b) \\
& H_{x}{ }^{\prime}=-j\left(E_{o} / c\right)(\lambda / 2 b) \sin \left(K^{1 / 2} \pi x / a\right) \cos (\pi y / b) \\
& H_{y}{ }^{\prime}=j\left(E_{o} / c\right)(\lambda / 2 a) \cos \left(K^{1 / 2} \pi x / a\right) \sin (\pi y / b)
\end{aligned}
$$

The energy density in the electric field in the dielectric $D^{\cdot} \mathrm{E}^{\prime} / 8 \pi$ $=E_{0}^{2} / 8 \pi$, the same as in the vacuum.

2. Cavity with quarter wave plate spaced $\lambda / 4$ from the side wall. ( see figure 4 )

Equation (1; is still applicable.

Equations (2) to (5) apply for $-\mathrm{a}<\mathrm{x}<0$

Equations (6) to (8) apply for $0<x<a \mathrm{~K}^{-1 / 2 / 2}$

$$
\begin{aligned}
& \text { At } \mathrm{x}=\mathrm{a} \mathrm{K}^{-1 / 2 / 2}, \mathrm{H}_{\mathrm{y}}^{\prime}=\mathrm{H}_{\mathrm{y}}^{\mathrm{n}}=0 \text {, } \\
& E_{z}^{\prime}=E_{z}^{n}=E_{0} K^{-1 / 2} \sin (\pi y / b) \text {, and } \\
& H_{x}^{\prime}=H_{x}^{n}=-j K^{-1 / 2\left(E_{0} / c\right) \lambda / 2 b \cos (\pi y / b)}
\end{aligned}
$$




$$
\begin{aligned}
& \text { For } a \mathrm{~K}^{-1 / 2}<\mathrm{x}<\mathrm{a}\left(1+\mathrm{K}^{-1 / 2) / 2}\right. \\
& E^{\prime \prime}{ }_{Z}=K^{-1 / 2} E_{0} \sin \left(\pi x / 2 a-K^{-1 / 2}\right) \sin (\pi y / b)
\end{aligned}
$$

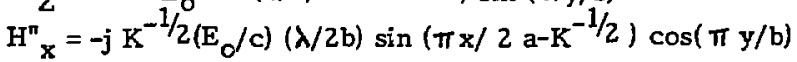

$$
\begin{aligned}
& H^{\prime \prime}{ }_{y}=j K^{-1 / 2}\left(E_{o} / c\right)(\lambda / 2 a) \cos \left(\pi x / 2 a-K^{-1 / 2}\right) \sin (\pi y / b)
\end{aligned}
$$

\section{B. Comparativie wall losses:}

According to Maxwells equation $\nabla \times H=i+\partial D / \partial t$, where $i$ is the current density. At the walls $D=K E \equiv 0$, so $\nabla \times H=i$, and losses $\sim \mathrm{I}^{2} \mathrm{r} \sim \mathrm{H}^{2} \mathrm{R}$, where $R$ is the surface resistance. Taking the explicit expressions for the $E$ and $H$ fields from section $A$ above, for the cavity shown in figure 4, we find at wall $W_{1}$,

$$
\begin{aligned}
E_{z}=H_{x_{b}}=0, H_{y} & =j\left(E_{o} / c\right)(\lambda / 2 a) \sin (\pi y / b) . \text { The loss } \\
L_{1} \sim \int_{0}^{H^{2} R d y} & =-\left(E_{o} / c\right)^{2}(\lambda / 2 a)^{2} b / 2 \\
& =C b / a^{2} \quad \text { where } C=E_{0}^{2} \lambda^{2} / 8 c^{2} .
\end{aligned}
$$

Let us consider a cavity with the design shown in figure 5 . The field and current distributions can be obtained by stacking a number $(\mathrm{N})$ of the cavities shown in figure 3 (removing walls $\mathrm{w}_{1}$ and $\mathrm{w}_{2}$ ) and adding cavities shown in figure 4 at each end (omitting wall $W_{1}$ ). Therefore, we may calculate the losses in the walls using the $E$ and $H$ field expressions from section $A$. The results are:

$\begin{array}{cccc}\text { Wall } & \mathrm{W}_{5} & \mathrm{~W}_{3} & \mathrm{w}_{4} \\ \text { Loss } & \mathrm{L}_{1} / \mathrm{K} & \mathrm{L}_{1}(\mathrm{a} / \mathrm{b})^{3} & \mathrm{~L}_{1}(\mathrm{a} / \mathrm{b})^{3} \mathrm{~K}^{-3 / 2}\end{array}$

The losses in walls $W_{4}$ (and in $W_{5}$ and $W_{6}$ ) can be neglected. So, for a cavity with overall dimensions of several wavelengths such as shown in figure 5 , the losses in the $2 \mathrm{~N}$ side walls $\mathrm{W}_{3}$ will be $\mathrm{N} \mathrm{K}(\mathrm{a} / \mathrm{b})^{3}$ of the losses in the two walls $\mathrm{W}_{5} \cdot \mathrm{N}$ is approximately equal to $\mathrm{b} / \mathrm{a}$, so losses in the side walls $\mathrm{W}_{3}$ will be smaller than in walls $W_{7}$ so long as $\mathrm{N}$ is greater than $\mathrm{K}^{1 / 2}$. For sapphire $\mathrm{K}=13$, so we require $N \geqslant 4$. Therefore, in a $25 \mathrm{~cm} \times 25 \mathrm{~cm}$ cavity, losses in the walls $W_{3}$ will be less than the losses in walls $W_{7}$ for frequenceis above $3 \mathrm{GHz}$. 


\section{Appendix IV}

\section{Mode Dependence of The Effective Volume of a Cavity}

\section{A. Einpty Rectangular Cavity:}

In the case of an empty rectangular cavity of dimensions $a x b y$, the amplitude for axion-photon conversion $A_{a}$ as given by Sikivie (private communication) (ignoring the time factor and taking the $p=0$ mode in the $d$ direction along $B_{0}$ ), is proportional to:

$$
\begin{aligned}
& \int_{V} E B d V=B_{0} E_{0} d \int_{0}^{d} \sin (n x / a) d x \int_{0}^{b} \sin (m y / b) d y \\
& =B_{0} E_{0} d(2 a / n \pi)(2 b / m \pi) \text { (if } n \text { and } m \text { are odd.) } \\
& =\left(4 / \pi^{2}\right) B_{0} \cdot E_{0} \cdot V \cdot(I / n m)
\end{aligned}
$$

The EM field energy of a photon

$$
\begin{aligned}
\text { hf } & =(2 / 8 \pi) \int_{V} E^{2} d V \\
& =(1 / 4 \pi) d \int_{0}^{2} \sin ^{2}(n \pi x / a) d x \int_{0}^{b} \sin ^{2}(m \pi y / b) d y
\end{aligned}
$$

but $\sin ^{2} x=1 / 2-1 / 2 \cos 2 x$, So, the energy of a photon

$$
\mathrm{hf}=\mathrm{E}_{\mathrm{o}}{ }^{2} \mathrm{~V} / 16 \pi \text {, and transposing, } \mathrm{E}_{0}{ }^{2}=16 \pi \mathrm{hf} / \mathrm{V}
$$

So, $P_{\partial \alpha} \sim A^{2}{ }_{a \gamma} \sim B_{0}^{2} E_{0}^{2} V^{2} / n^{2} m^{2} \sim h f B_{0}^{2} V / n^{2} m^{2}$ 


\section{B. Cavity with Half Wave Dielectric Plates}

The field distribution in a cavity with spaced half wave dielectric plates (figure 5 ) is a periodic repetition of the field in a cavity with one dielectric plate shown in figure 3. The $E$ and $H$ fields for this cavity are given in Appendix III $A(1)$. In this case $A_{a}$ is proportional to :

$$
\begin{aligned}
& \int_{V} E \mathrm{~B} d V=B_{0} E_{0} d \int_{0}^{b} \sin (m y / b) d y\left[\int_{-a}^{0} \sin (x / a) d x+\int^{a K^{-1 / 2}} K^{-1 / 2} \sin \left(K^{1 / 2} x / a\right) d x\right] \\
& =-\left(4 / \pi^{2}\right) B_{0} E_{0} \text { abd }\left(1-K^{-1}\right)
\end{aligned}
$$

Now $V=\operatorname{abd}\left(1+K^{-1 / 2}\right)$, and $\left(1-K^{-1}\right)=\left(1-K^{-1 / 2}\right)\left(1+K^{-1 / 2}\right)$, so

$$
\int_{V} E \mathrm{~B} d V=-\left(4 / \pi^{2}\right) B_{0} E_{0} V\left(1-K^{-1 / 2}\right)
$$

From Appendix III A (1), the energy density is the same in the dielectric as in the vacuum. Therefore the EM field energy of a photon:

$$
\mathrm{hf}=(2 / 8 \pi) \int_{V} E \mathrm{D} d V=E_{0}^{2} \mathrm{~V} / 16 \pi \text {. Transposing, } E_{0}^{2}=16 \mathrm{hf} / \mathrm{V} \text { as in }
$$

So $P_{\text {a }} \sim A_{\text {a }}^{2} \sim B_{0}{ }^{2} E_{o}{ }^{2} V^{2}\left(1-K^{-1 / 2}\right)^{2} \sim h_{f B}{ }^{2} V\left(1-K^{-1 / 2}\right)^{2}$. The difference between this result and the calculation for an empty cavity given in section $A$ is that the factor $1 / \mathrm{n}^{2} \mathrm{~m}^{2}$ is replaced by $\left(1-\mathrm{K}^{-1 / 2}\right)^{2}$ =

The factor $\left(1-K^{-1 / 2}\right)^{2}$ is about 0.52 for $K=13$, and 0.47 for $K=10$, so the power produced in a cavity using half wave dielectric phasing plates is larger than the power in an empty cavity with $n$ or m greater than one. 


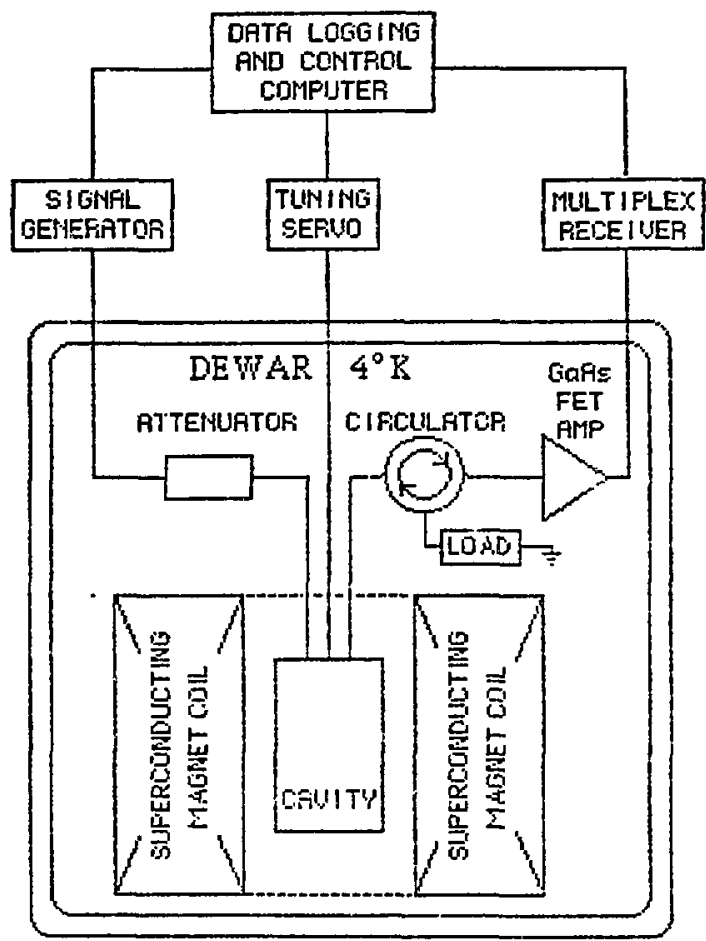

FIGURE 1

Ahion detection system 


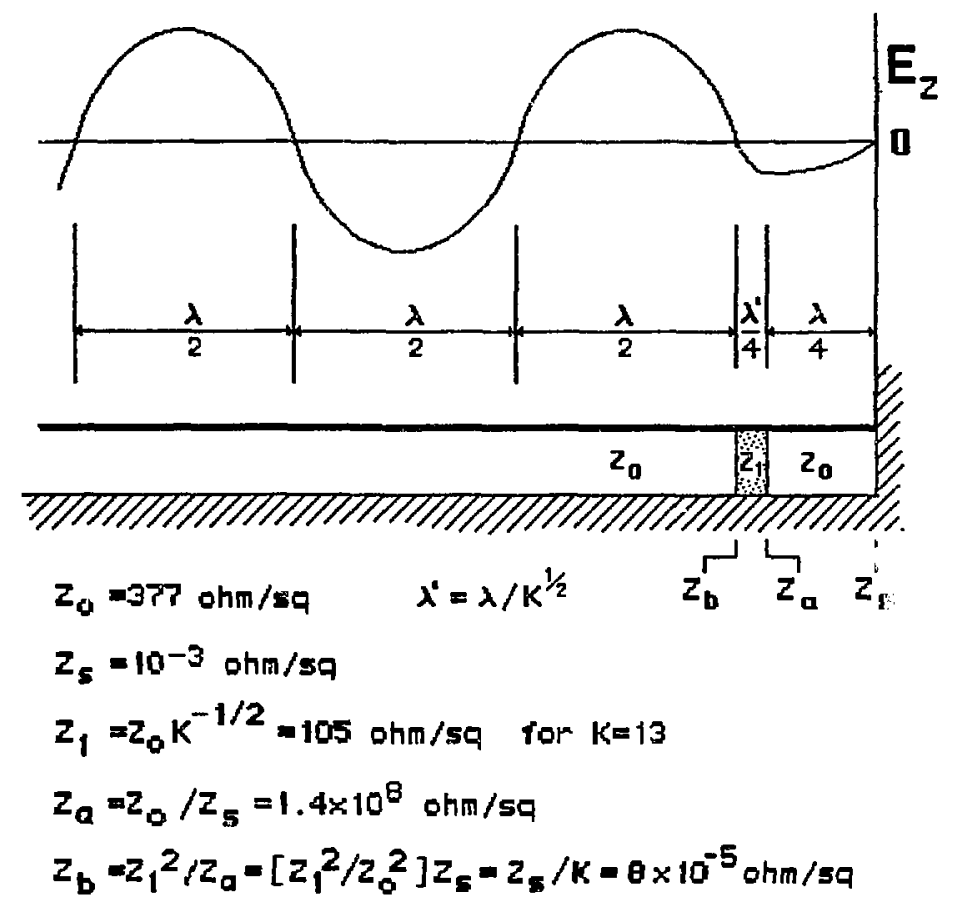

FIGURE 2

Transmission line equivelent

of quarter waue plate

tu reduce walf loss 


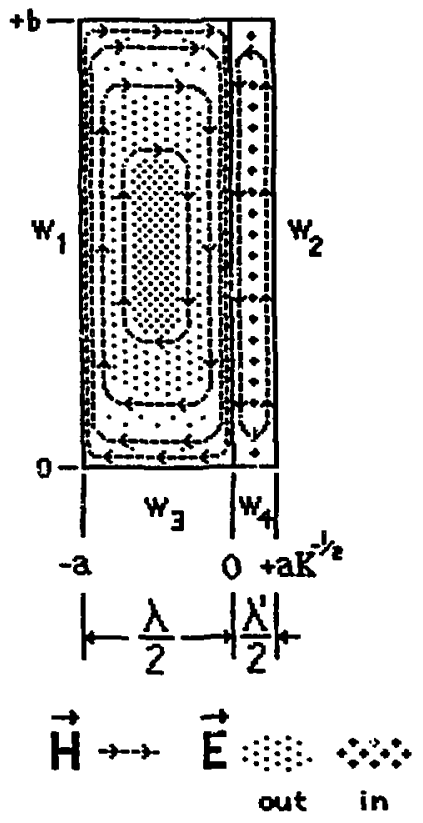

FIGURE 3

\section{Cavity with half wave} dielectric plate

(see appendix I)

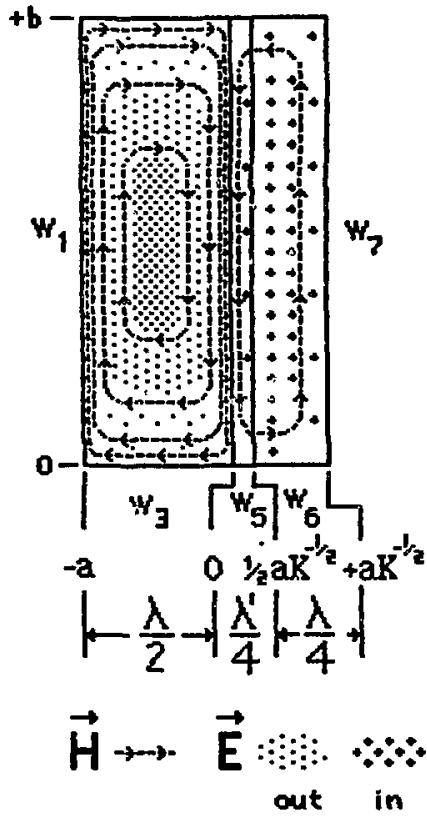

FIGURE 4

Cavity with quarter wave dielectric plate

(see appandix 1) 


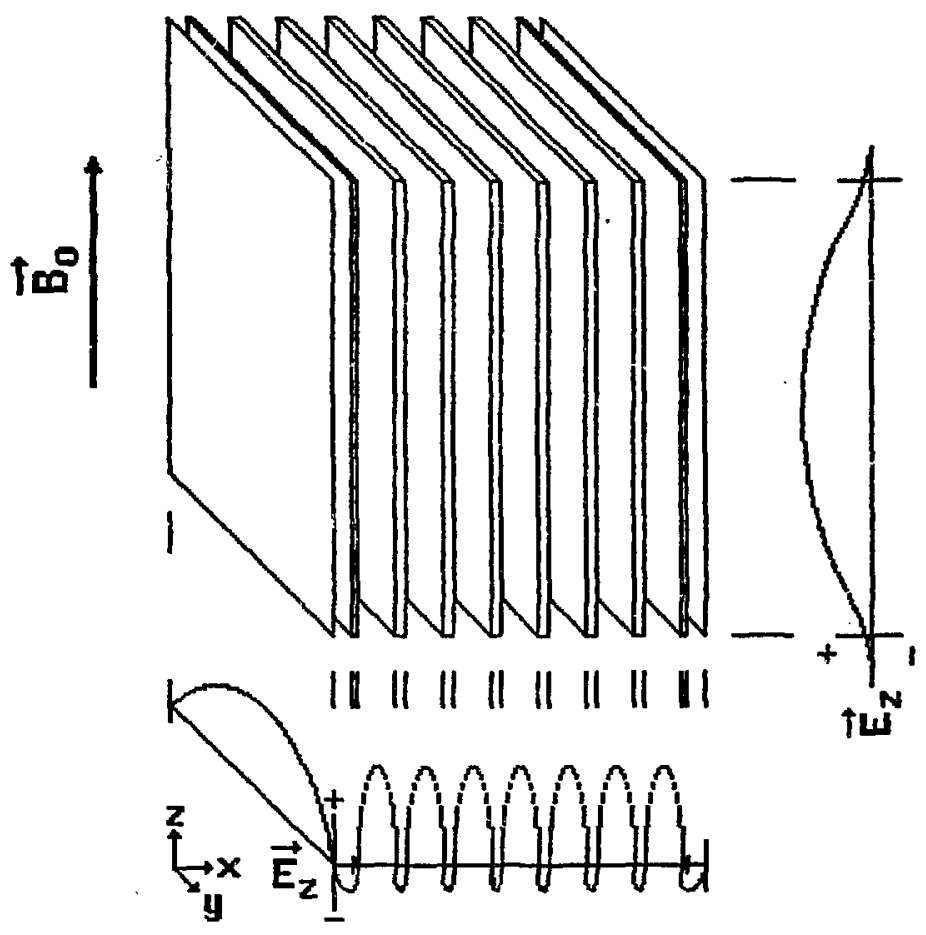

Figure 5A

Cauity with Half Have Dielectric Plates 


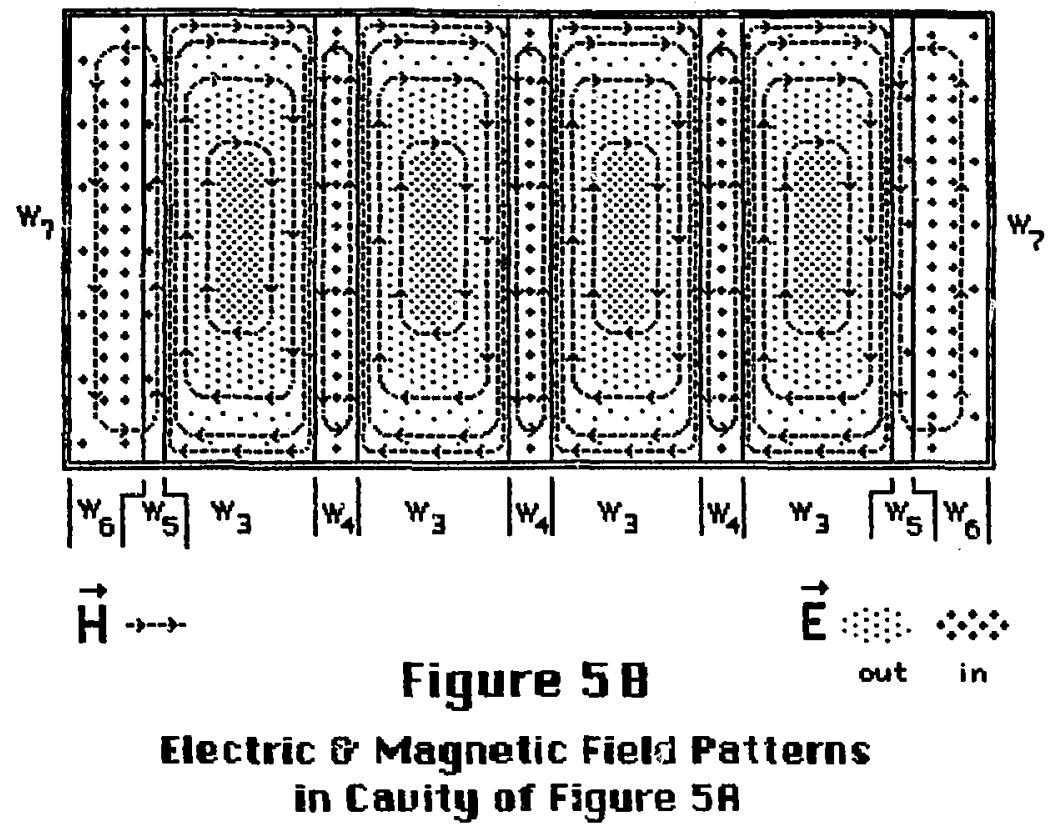




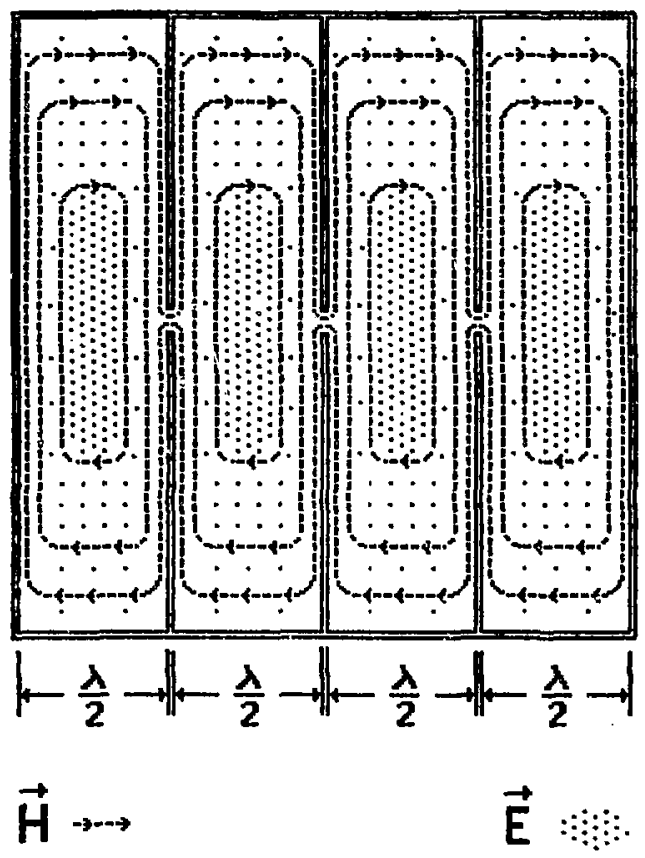

FIGURE 6

Cauity with meta: jaffles with half wave specing 
This report was done with support from the Department of Energy. Any conclusions or opinions expressed in this report represent solely those of the author(s) and not necessarity those of The Regents of the Liniversity of California, the Lawrence Berkeley Laboratory or the Depariment of Energy.

Reference to a company or product name does not imply approval or recommendation of the product by the University of Califomia or the U.S. Department of Energy to the exclusion of others that may be suitable. 